\title{
ZERO SETS AND EXTENSIONS OF BOUNDED HOLOMORPHIC FUNCTIONS IN POLYDISCS
}

\author{
P. S. CHEE
}

\begin{abstract}
A sufficient condition for a hypersurface in a polydisc $U^{n}$ to be the zero set of an $H^{\infty}\left(U^{n}\right)$ function is proved. This strengthens a result of Zarantonello and generalizes a result of Rudin. Using this result and a result of Andreotti and Stoll, a partial extension of Alexander's theorem on extension of bounded holomorphic functions from a hypersurface of $U^{n}$ to $U^{n}$ is obtained. Finally, a generalization of Cima's extension theorem for $\boldsymbol{H}^{p}$ functions is given.
\end{abstract}

1. Introduction. Let $U^{n}$ be the open unit polydisc in the space $\mathbf{C}^{n}$ of $n$ complex variables. Let $N\left(U^{n}\right)$ and $H^{p}\left(U^{n}\right), 0<p \leqslant \infty$, denote the Nevanlinna and Hardy classes respectively. (For definitions, see [6].) Rudin [6, Theorem 4.8.3] first gave a sufficient condition for the zero sets of $H^{\infty}\left(U^{n}\right)$. Later, Zarantonello [9] gave a sufficient condition for the zero sets of $N\left(U^{n}\right)$. In this paper, we show that Zarantonello's condition is also a sufficient condition for the zero sets of $H^{\infty}\left(U^{n}\right)$. This generalizes both the results of Rudin and of Zarantonello, and answers a question raised at the end of [9].

Next we consider the extension of bounded holomorphic functions from a hypersurface of $U^{n}$ to $U^{n}$. This problem has been discussed by Alexander [2] and Andreotti and Stoll [3]. Using the above result, and a theorem of Andreotti and Stoll, we give a partial extension of Alexander's extension theorem.

Finally, a generalization of the extension theorem of Cima [4] is given in $\S 5$.

2. Notations. The following notations will be used. If $0<r \leqslant 1$, then $U(r)=\{z \in \mathbf{C}:|z|<r\}$; as usual, we write $U$ for $U(1)$. If $0<r<s$, then $Q(r, s)=\{z \in \mathbf{C}: r<|z|<s\}$. The unit circle $\{z \in \mathbf{C}:|z|=1\}$ is denoted by $T$ and the unit $n$-torus by $T^{n}=T \times \cdots \times T$ ( $n$ copies). $T^{n}$ is the distinguished boundary of $U^{n}$.

If $\Omega$ is an open subset of $\mathbf{C}^{n}$, then $H(\Omega)$ denotes the set of all holomorphic functions in $\Omega$, and $H^{\infty}(\Omega)$ denotes the subset of all bounded ones. The zero set of $f \in H(\Omega)$ is $Z(f)=f^{-1}(0)$.

A subvariety $E$ of $U^{n}$ is said to satisfy Zarantonello's condition if

Received by the editors November 10, 1975 and, in revised form, December 15, 1975.

AMS (MOS) subject classifications (1970). Primary 32A30, 32D20.

Key words and phrases. Polydiscs, bounded holomorphic functions, Hardy classes, zero sets, extension of bounded holomorphic functions, removable singularities, second Cousin problem. 
there exists a constant $0<r<1$ and a continuous function $\eta=[r, 1) \rightarrow[r, 1)$ such that

$$
\left|z_{n}\right| \leqslant \eta\left(\frac{\left|z_{1}\right|+\cdots+\left|z_{n-1}\right|}{n-1}\right)
$$

for all $z=\left(z_{1}, \ldots, z_{n}\right) \in E \cap Q^{n}(R, 1)$.

3. Zero sets of $H^{\infty}\left(U^{n}\right)$. The following theorem gives a strengthening of the result of [9]. The proof is similar to that of [9].

THEOREM 3.1. Suppose $n \geqslant 2$ and $f \in H\left(U^{n}\right)$. If $E=Z(f)$ satisfies Zarantonello's condition (2.1), then there exists an $F \in H^{\infty}\left(U^{n}\right)$ such that $f=F e^{h}$ for some $h \in H\left(U^{n}\right)$.

Proof. Choose an $r \in(0,1)$ and a continuous function $\eta:[r, 1) \rightarrow[r, 1)$ such that $(2.1)$ is satisfied. Fix $r^{\prime} \in(r, 1)$. Choose $c$ such that

$$
1>c>c^{\prime}=\sup \left\{\eta(x): r \leqslant x \leqslant 1-\left(1-r^{\prime}\right) /(n-1)\right\} \text {. }
$$

Let

$$
\begin{aligned}
V_{i} & =U^{i-1} \times U\left(r^{\prime}\right) \times U^{n-i}, \quad 1 \leqslant i \leqslant n-1, \\
V_{n} & =Q^{n-1}(r, 1) \times U .
\end{aligned}
$$

Further, let

$$
Q_{i}=Q^{i-1}(r, 1) \times Q\left(r, r^{\prime}\right) \times Q^{n-i-1}(r, 1) \times Q(c, 1), \quad 1 \leqslant i \leqslant n-1 \text {. }
$$

Note that

$$
\begin{aligned}
V_{i} \cap V_{k}=U^{i-1} \times U\left(r^{\prime}\right) \times U^{k-i-1} \times U\left(r^{\prime}\right) \times U^{n-k}, & 1 \leqslant i<k \leqslant n-1,
\end{aligned}
$$

and

$$
V_{i} \cap V_{n}=Q^{i-1}(r, 1) \times Q\left(r, r^{\prime}\right) \times Q^{n-i-1}(r, 1) \times U, \quad 1 \leqslant i \leqslant n-1,
$$

are polydomains whose distinguished boundaries are products of the boundaries of the factors. In particular, the distinguished boundary of $V_{i} \cap$ $V_{n}$ is contained in that of $Q_{i}, 1 \leqslant i \leqslant n-1$.

The polydomains $\left\{V_{i}: 1 \leqslant i \leqslant n\right\}$ form an open cover of $U^{n}$. They can be enlarged to form an open cover of $\bar{U}^{n}$ such that the intersection of the enlargement of $V_{i}$ with $U^{n}$ is $V_{i}$. We proceed to construct bounded Cousin data for the cover $\left\{V_{i}\right\}$ and then apply Stout's theorem [8].

Suppose $1 \leqslant i \leqslant n-1$. If $\left(z_{1}, \ldots, z_{n-1}\right) \in Q^{i-1}(r, 1) \times Q\left(r, r^{\prime}\right) \times$ $Q^{n-i-1}(r, 1)$ and $f\left(z_{1}, \ldots, z_{n-1}, z_{n}\right)=0$, then

$$
\left|z_{n}\right| \leqslant \eta\left(\frac{\left|z_{1}\right|+\cdots+\left|z_{n-1}\right|}{n-1}\right) \leqslant c^{\prime}<c .
$$

Hence $\operatorname{dist}\left(Z(f), Q_{i}\right)>0$. It follows from Rudin's theorem [6, Theorem 4.8.3] (applied to the restriction of $f$ to $V_{i}$ ) that there exists an $F_{i} \in H^{\infty}\left(V_{i}\right)$ such that $F_{i} f^{-1}$ is an invertible element of $H\left(V_{i}\right)$, and $F_{i}^{-1}$ is bounded in $Q_{i}$. 
Next, we show that the same is true in $V_{n}$. Fix $z^{\prime} \in Q^{n-1}(r, 1)$. Then $f\left(z^{\prime}, \cdot\right)$ has finitely many zeros in $U$, by the hypothesis on $f$. Let these zeros be $\alpha_{1}\left(z^{\prime}\right), \ldots, \alpha_{k}\left(z^{\prime}\right)$, listed according to multiplicities. Put

$$
F_{n}(z)=\prod_{1}^{k}\left(z_{n}-\alpha_{i}\left(z^{\prime}\right)\right), \quad z=\left(z^{\prime}, z_{n}\right) \in V_{n} .
$$

Then $k$ is independent of $z^{\prime}$ and $F_{n} \in H\left(V_{n}\right)$ (see [9, p. 312]). Clearly, $F_{n}$ is bounded and has the same zeros as $f$ in $V_{n}$. Since $\operatorname{dist}\left(Z(f), Q_{i}\right) \geqslant c-c^{\prime}>$ 0 , it follows that $\left|F_{n}\right| \geqslant\left(c-c^{\prime}\right)^{k}>0$ in $Q_{i}$. Hence $F_{n}^{-1}$ is bounded in $Q_{i}$, $1 \leqslant i \leqslant n-1$.

Since, for all $i, F_{i} f^{-1}$ is a zero-free holomorphic function in $V_{i}$, so is $F_{i} F_{k}^{-1}$ in $V_{i} \cap V_{k}$, for all $i, k$. We claim that $F_{i} F_{k}^{-1}$ is bounded in $V_{i} \cap V_{k}, 1 \leqslant i$, $k \leqslant n$.

Suppose $1 \leqslant i<k \leqslant n-1$. Then $F_{i} F_{k}^{-1}$ is holomorphic in $V_{i} \cap V_{k}$ and is bounded in $Q_{k}$. The distinguished boundary of $V_{i} \cap V_{k}$ is contained in $\bar{Q}_{k}$. Hence, by the maximum modulus theorem, $F_{i} F_{k}^{-1}$ is bounded in $V_{i} \cap V_{k}$. Similarly, $F_{k} F_{i}^{-1}$ is bounded in $V_{i} \cap V_{k}$.

Suppose $1 \leqslant i \leqslant n-1$. Then $F_{i} F_{n}^{-1}$ is holomorphic in $V_{i} \cap V_{n}$ and is bounded in $Q_{i}$. Since the distinguished boundary of $V_{i} \cap V_{n}$ is contained in that of $Q_{i}$, the maximum modulus theorem again shows that $F_{i} F_{n}^{-1}$ is bounded in $V_{i} \cap V_{n}$. Similarly, $F_{n} F_{i}^{-1}$ is bounded in $V_{i} \cap V_{n}$.

Hence, for all $i, k, F_{i} F_{k}^{-1}$ is an invertible element of $H^{\infty}\left(V_{i} \cap V_{k}\right)$. By Stout's theorem [8], there exists an $F \in H^{\infty}\left(U^{n}\right)$ such that $F F_{i}^{-1}$ is an invertible element of $H^{\infty}\left(V_{i}\right), 1 \leqslant i \leqslant n$. Since $F_{i} f^{-1}$ is an invertible element of $H\left(V_{i}\right)$, it follows that $F f^{-1}$ is zero-free in $V_{i}, 1 \leqslant i \leqslant n$. Since $\left\{V_{i}: 1 \leqslant i\right.$ $\leqslant n\}$ covers $U^{n}, F f^{-1}$ is zero-free in $U^{n}$ and so there exists an $h \in H\left(U^{n}\right)$ such that $f=\mathrm{Fe}^{h}$.

Remark. For later applications, we note that for each $i$, there exists $\psi_{i} \in H^{\infty}\left(V_{i}\right)$ such that $\psi_{i}^{-1} \in H^{\infty}\left(V_{i}\right)$ and $F=F_{i} \psi_{i}$. Hence $F^{-1}$ is bounded in $Q_{i}, 1 \leqslant i \leqslant n-1$.

4. Extensions of bounded holomorphic functions. Using the results of $\$ 3$ and of Andreotti and Stoll [3], we can now give a partial extension of the result of Alexander [2].

THEOREM 4.1. Let $E$ be a subvariety of $U^{n}, n \geqslant 2$, of pure dimension $n-1$, satisfying condition (2.1) and the following condition of Alexander:

$$
\begin{aligned}
& \text { there exists } a \delta>0 \text { such that if } r \text { is as in }(2.1), 1 \leqslant i \leqslant n \text {, } \\
& \left(z^{\prime}, \alpha, z^{\prime \prime}\right) \text { and }\left(z^{\prime}, \beta, z^{\prime \prime}\right) \in E \cap\left[Q^{i-1}(r, 1) \times U \times\right.
\end{aligned}
$$$$
\left.Q^{n-i}(r, 1)\right] \text {, and } a \neq b \text {, then }|\alpha-\beta| \geqslant \delta \text {. }
$$

Then for all bounded holomorphic functions $g$ on $E$, there exists a bounded holomorphic function $G$ in $U^{n}$ such that $G=g$ on $E$.

Proof. Fix $r^{\prime} \in(r, 1)$. Let $c, V_{i}, Q_{i}$ be as defined in §3. It was shown in [2] that there exists $f \in H\left(U^{n}\right)$ such that $E=Z(f)$ and $\partial f / \partial z_{i} \neq 0$ on $E \cap$ 
$\left[Q^{i-1}(r, 1) \times U \times Q^{n-i}(r, 1)\right], 1 \leqslant i \leqslant n$. By Theorem 3.1, there exists $F \in$ $H^{\infty}\left(U^{n}\right)$ such that $f=F e^{u}$ for some $u \in H\left(U^{n}\right)$. Hence $\partial F / \partial z_{i} \neq 0$ on $E \cap\left[Q^{i-1}(r, 1) \times U \times Q^{n-i}(r, 1)\right], 1 \leqslant i \leqslant n$. By the remark at the end of \$3, there exists $\psi \in H^{\infty}\left(V_{n}\right)$ such that $\psi^{-1} \in H^{\infty}\left(V_{n}\right)$ and $F=F_{n} \psi$. By condition (4.1) and definition (3.1) of $F_{n}$, it follows that $\left|\partial F_{n} / \partial z_{n}\right|$ is bounded from 0 on $E \cap V_{n}$. Since $\partial F / \partial z_{n}=\psi \partial F_{n} / \partial z_{n}$ on $E \cap V_{n}$, it follows that there exists $\varepsilon>0$ such that $\left|\partial F / \partial z_{n}\right| \geqslant \varepsilon$ on $E \cap V_{n}$.

Let $g \in H^{\infty}(E)$. For $1 \leqslant i \leqslant n-1$, it follows from Alexander's theorem [2] that there exists $g_{i} \in H^{\infty}\left(V_{i}\right)$ such that $g=g_{i}$ on $E \cap V_{i}$. We show that the same is true in $V_{n}$.

By Cartan's theorem (see [6, Theorem 7.1.2]) there exists $\phi \in H\left(U^{n}\right)$ such that $\phi=g$ on $E \cap V_{n}$. Let $\left(z^{\prime}, z_{n}\right) \in V_{n}$, and let

$$
s=\eta\left(\frac{\left|z_{1}\right|+\cdots+\left|z_{n-1}\right|}{n-1}\right) \text {. }
$$

Choose a positively oriented circle $\gamma$ with center 0 , lying in $Q(s, 1)$ and enclosing $z_{n}$. Put

$$
h\left(z^{\prime}, z_{n}\right)=\frac{1}{2 \pi i} \int_{\gamma} \frac{\phi\left(z^{\prime}, \zeta\right)}{F\left(z^{\prime}, \zeta\right)} \frac{d \zeta}{\zeta-z_{n}} .
$$

Then $h$ is independent of the choice of $\gamma$ and $h \in H\left(V_{n}\right)$. Let $g_{n}=\phi-h F$. Then $g_{n}=g$ on $E \cap V_{n}$. We claim that $g_{n} \in H^{\infty}\left(V_{n}\right)$. Let $\gamma_{1}, \ldots, \gamma_{k}$ be small circles about the zeros $\alpha_{1}\left(z^{\prime}\right), \ldots, \alpha_{k}\left(z^{\prime}\right)$ of $F\left(z^{\prime}, \cdot\right)$. Then by the computation given in [2, p. 488],

$$
(\phi-h F)\left(z^{\prime}, z_{n}\right)=\sum_{j=1}^{k} \frac{g\left(z^{\prime}, \alpha_{j}\left(z^{\prime}\right)\right)}{\left(\partial F / \partial z_{n}\right)\left(z^{\prime}, \alpha_{j}\left(z^{\prime}\right)\right)} \cdot \frac{F\left(z^{\prime}, z_{n}\right)}{z_{n}-\alpha_{j}\left(z^{\prime}\right)} .
$$

Since $F=F_{n} \psi$, each $F\left(z^{\prime}, z_{n}\right) /\left(z_{n}-\alpha_{j}\left(z^{\prime}\right)\right)$ is bounded on $V_{n}$. Since $\left|\partial F / \partial z_{n}\right|$ $\geqslant \varepsilon$ on $E \cap V_{n}$, and each $\left(z^{\prime}, \alpha_{j}\left(z^{\prime}\right)\right) \in E \cap V_{n}$, it follows that $g_{n}=\phi-h F$ is bounded on $V_{n}$.

For $1 \leqslant i<k \leqslant n$, and $z=\left(z^{\prime}, z_{n}\right) \in V_{i} \cap V_{k}$, put

$$
a_{i k}(z)=\frac{1}{2 \pi i} \int_{\gamma} \frac{g_{i}\left(z^{\prime}, \zeta\right)-g_{k}\left(z^{\prime}, \zeta\right)}{F\left(z^{\prime}, \zeta\right)} \frac{d \zeta}{\zeta-z_{n}},
$$

where $\gamma$ is a positively oriented circle with center 0 and radius $>\max \left(c,\left|z_{n}\right|\right)$. Then $a_{i k}$ is independent of the choice of $\gamma$ and $a_{i k} \in H\left(V_{i} \cap V_{k}\right)$.

Suppose $1 \leqslant i<k \leqslant n-1$. Since $F=0$ on $E$ and $\partial F / \partial z_{n} \neq 0$ on $E \cap$ $V_{n}, F\left(z^{\prime}, \cdot\right)$ has simple zeros. Since $g_{i}-g_{k}=0$ on $E \cap V_{i} \cap V_{k}$, it follows that $\left(g_{i}-g_{k}\right) F^{-1}$ is holomorphic in $V_{i} \cap V_{k} \cap V_{n}$. Therefore, by Cauchy's integral formula, $a_{i k}=\left(g_{i}-g_{k}\right) F^{-1}$ in $V_{i} \cap V_{k} \cap V_{n}$. Since this is an open subset of $V_{i} \cap V_{k}$ which is connected, we must have

$$
g_{i}-g_{k}=a_{i k} F \text { in } V_{i} \cap V_{k} \text {. }
$$

Since $F^{-1}$ is bounded in $Q_{i}$, and the distinguished boundary of $V_{i} \cap V_{k}$ is contained in $\bar{Q}_{i}, a_{i k} \in H^{\infty}\left(V_{i} \cap V_{k}\right)$ by the maximum modulus theorem. 
Suppose $1 \leqslant i \leqslant n-1$. Then by the reasons given above, $\left(g_{i}-g_{n}\right) F^{-1}$ is holomorphic in $V_{i} \cap V_{n}$. So by Cauchy's integral formula,

$$
g_{i}-g_{n}=a_{i n} F \text { in } V_{i} \cap V_{n} .
$$

Since $F^{-1}$ is bounded on $Q_{i}$, and the distinguished boundary of $V_{i} \cap V_{n}$ is contained in $\bar{Q}_{i}$, it follows by the maximum modulus theorem that $a_{i n} \in$ $H^{\infty}\left(V_{i} \cap V_{n}\right)$.

Now we conclude by Theorem 2.8 of [3] that there exists a $G \in H^{\infty}\left(U^{n}\right)$ such that $G=g$ on $E$.

RemarK. Alexander has shown that if $E$ satisfies Rudin's condition that $\operatorname{dist}\left(E, T^{n}\right)>0$, together with condition (4.1), then there exists a bounded linear operator $T: H^{\infty}(E) \rightarrow H^{\infty}\left(U^{n}\right)$ such that $T f=f$ on $E$.

It follows quite easily from the open mapping theorem that under the hypothesis of Theorem 4.1, there exists a constant $M$ such that every $f \in H^{\infty}(E)$ has an extension $F \in H^{\infty}\left(U^{n}\right)$ satisfying $\|F\|_{U^{n}} \leqslant M\|f\|_{E}$ (see [10, p. 517]). However, we do not know if the extension $F$ can be chosen to depend linearly on $f$.

5. Removable singularities. Let $E$ be a closed subset of $U^{n}$. For $0<p<\infty$, we say that $f \in H^{p}\left(U^{n}-E\right)$ if $f$ is holomorphic in $U^{n}-E$ and $|f|^{p}$ has an $n$-harmonic majorant in $U^{n}-E$. If $E$ is empty, this condition is equivalent to the usual one, namely,

$$
\sup _{0<r<1} \int_{T^{n}}|f(r w)|^{p} d m(w)<\infty
$$

where $m$ is the normalized Haar measure on $T^{n}$. (See [6, Chapter 3].) We wish to consider whether $E$ is a set of removable singularities of $f$.

For $n=1$, Parreau [5, p. 182] has proved that if $E$ has logarithmic capacity zero, then every $f \in H^{p}(U-E)$ can be extended to an $F \in H^{p}(U)$. For $n>1$, it is a result of Shiffman [7, Lemma 3] that every set $E$ with $(2 n-1)$-dimensional Hausdorff measure zero is removable for $f \in H^{\infty}\left(U^{n}\right)$. If $1 \leqslant p<\infty$, Cima [4] has shown that $E$ is removable if $E$ is a hypersurface of $U^{n}$ satisfying Rudin's condition: $\operatorname{dist}\left(E, T^{n}\right)>0$. We show that in Cima's result, Rudin's condition can be replaced by Zarantonello's. Furthermore, we only require $|f|^{p}$ to have an $n$-harmonic majorant in $U^{n}-E$ instead of an $R P$-majorant as in [4].

Theorem 5.1. Let $n \geqslant 2,0<p<\infty$. Suppose $E$ is a subvariety of $U^{n}$ of pure dimension $n-1$ satisfying condition (2.1). If $f \in H^{p}\left(U^{n}-E\right)$, then there exists an $F \in H^{p}\left(U^{n}\right)$ such that $F=f$ on $U^{n}-E$.

Proof. Since the case $\lim _{s \rightarrow 1} \eta(s)<1$ is covered by [4], we may assume that $\lim _{s \rightarrow 1} \eta(s)=1$.

Suppose $f \in H^{p}\left(U^{n}-E\right)$. We show first that $f$ extends to a holomorphic function in $U^{n}$. By Theorem 2.1, there exists $g \in H^{\infty}\left(U^{n}\right)$ such that $E=$ $Z(g)$. Let $a=\left(a_{1}, \ldots, a_{n}\right) \in E$. If $g\left(z^{\prime}, a_{n}\right) \neq 0$ as a function of $z^{\prime}$, then by the proof given in [4, p. 531], $f$ extends to a holomorphic function in $U^{n}$. If 
$g\left(z^{\prime}, a_{n}\right) \equiv 0$, then there exist a positive integer $\alpha$ and a function $g_{1} \in H\left(U^{n}\right)$ such that $g(z)=\left(z_{n}-a_{n}\right)^{\alpha} g_{1}(z)$, where $g_{1}\left(z^{\prime}, a_{n}\right) \neq 0$. Hence $Z(g)=\{z \in$ $\left.U^{n}: z_{n}=a_{n}\right\} \cup Z\left(g_{1}\right)$. Since by the proof given in [4, p. 531], $f$ extends holomorphically over both $\left\{z \in U^{n}: z_{n}=a_{n}\right\}$ and $Z\left(g_{1}\right)$, it follows that there exists $F \in H\left(U^{n}\right)$ such that $F=f$ on $U^{n}-E$.

To show that $F \in H^{p}\left(U^{n}\right)$, we note first that by the $n$-subharmonicity of $|F|^{p}$

$$
\sup _{0<r<1} \int_{T^{n}}|F(r w)|^{p} d m(w)=\sup _{0<s<1} \int_{T^{n}}\left|F\left(s w^{\prime}, t w_{n}\right)\right|^{p} d m(w),
$$

where $t=\frac{1}{2}(1+\eta(s)), \quad w=\left(w^{\prime}, w_{n}\right)=\left(w_{1}, \ldots, w_{n-1}, w_{n}\right)$. By hypothesis, there exists an $n$-harmonic function $u$ in $U^{n}-E$ such that $|F|^{p} \leqslant u$ in $U^{n}-E$. Hence

$$
\int_{T^{n}}\left|F\left(s w^{\prime}, t w_{n}\right)\right|^{p} d m(w) \leqslant \int_{T^{n}} u\left(s w^{\prime}, t w_{n}\right) d m(w) .
$$

It is therefore sufficient to show that the last integral is bounded as $s \rightarrow 1$.

For $r^{\prime} \in(r, 1)$, let $r_{1}=\max \left\{\eta(x): r \leqslant x \leqslant r^{\prime}\right\}$. Then $u$ is $n$-harmonic in the polyannulus $Q^{n-1}\left(r, r^{\prime}\right) \times Q\left(r_{1}, 1\right)$. If $r_{1}<t<1$, then by a well-known result (see e.g. [1, Chapter 5]), we have

$$
\int_{T} u\left(s w^{\prime}, t w_{n}\right) d m\left(w_{n}\right)=u_{1}\left(s w^{\prime}\right) \log t+v_{1}\left(s w^{\prime}\right)
$$

where $u_{1}$ and $v_{1}$ are $(n-1)$ harmonic in $Q^{n-1}\left(r, r^{\prime}\right)$. Repeated integration gives

$$
\int_{T^{n}} u\left(s w^{\prime}, t w_{n}\right) d m(w)=\sum_{i=0}^{n-1}\left(\alpha_{i} \log t+\beta_{i}\right)(\log s)^{i}
$$

where $\alpha_{i}, \beta_{i}$ are constants. Since both $s$ and $t$ are bounded from 0 and $\infty$, it follows that the right side of (5.1) is bounded as $s \rightarrow 1$. This completes the proof.

Acknowledgement. Part of this work was done while the author was attending the Summer Course in Complex Analysis (1975) at the International Centre for Theoretical Physics, Trieste, whose partial support is hereby acknowledged.

\section{REFERENCES}

1. L. V. Ahlfors, Complex analysis: An introduction to the theory of analytic functions of one complex variable, 2nd ed., McGraw-Hill, New York, 1966. MR 32 \# 5844.

2. H. Alexander, Extending bounded holomorphic functions from certain subvarieties of a polydisc, Pacific J. Math. 29 (1969), 485-490. MR 39 \# 5822.

3. A. Andreotti and W. Stoll, The extension of bounded holomorphic functions from hypersurfaces in a polycylinder, Rice Univ. Studies 56 (1970), 199-222. MR 43 \#485.

4. J. A. Cima, An extension theorem for $H^{p}$ functions, Proc. Amer. Math. Soc. 42 (1974), 529-532. MR 48 \#4349.

5. M. Parreau, Sur les moyennes des fonctions harmoniques et analytiques et la classification des surfaces de Riemann, Ann. Inst. Fourier (Grenoble) 3 (1951), 103-197. MR 14, 263.

6. W. Rudin, Function theory in polydiscs, Benjamin, New York, 1969. MR 41 \#501. 
7. B. Shiffman, On the removal of singularities of analytic sets, Michigan Math. J. 15 (1968), 111-120. MR 37 \# 464.

8. E. L. Stout, The second Cousin problem with bounded data, Pacific J. Math. 26 (1968), 379-387. MR 38 \#3467.

9. S. E. Zarantonello, The multiplicative Cousin problem and a zero set for the Nevanlinna class in the polydisc, Trans. Amer. Math. Soc. 200 (1974), 291-313. MR 50 \# 7569.

10. H. S. Shapiro and A. L. Shields, On some interpolation problems for analytic functions, Amer. J. Math. 83 (1961), 513-532. MR 24 \# A3280.

Department of Mathematics, University of Malaya, Kuala Lumpur, Malaysia 